\title{
INSIGHT INTO THE APPLICATION OF COMPUTED TOMOGRAPHY TO BUILDING MATERIALS RESEARCH
}

\author{
Osburg, Andrea; Hadlich, Christiane; Vogt, Franziska \\ Chair of Building Chemistry and Polymer Materials, F.A. Finger-Institute for \\ Building Material Engineering, Bauhaus-Universität Weimar, Germany.
}

\begin{abstract}
Computed tomography is presented as a highly suitable non-destructive method to study the micro structure, behaviour and other characteristics of different objects and materials for the use in the field of civil engineering. The method and workflow are introduced and its potential is shown on selected examples.

Besides the many advantages of CT in civil engineering, there are some obstacles that need to be overcome. The selected examples present two of the main obstacles and offer solutions for how to deal with them.

The first discussed aspect is the correlation between sample size and achievable voxel size. The voxel size determines the size of details that can be detected by the CTsystem. It is often necessary to take small sub-samples, however this may limit the representativeness of the sample. The second discussed aspect is segmentation of CT datasets. Often artefacts and low contrast complicate the process of segmentation. Different algorithms can be used to overcome this obstacle; two possible approaches are presented.
\end{abstract}

Keywords: Computed tomography, building materials.

\section{INTRODUCTION}

To study the micro structure, behaviour and other characteristics of different objects and materials often non-destructive methods are needed which also enable the 3dimensional imaging of the specimen. In many cases of such scientific issues computed tomography is a highly suitable technique. Computed tomography (CT) is based on X-ray and is used in different scientific sectors like medical science, biology, mechanical engineering and archaeology. [1-12] Also in the field of material research this method gets more and more important.

There are different configurations of CT-systems available. The configuration significantly determines the kind of materials and the kind of research questions that can be analysed.

This paper introduces the possibilities and limitations of the sub- $\mu$-CT-system „nanotom $m$ research I edition“ constructed by GE. The potentials of the application 
of this system for materials research and other aspects of civil engineering are demonstrated by selected examples.

\section{METHOD AND WORKFLOW}

Computed tomography is a non-destructive imaging method based on twodimensional X-ray images. By means of different algorithms image stacks consisting of two-dimensional X-ray images are reconstructed to a three-dimensional volume data set.

The system mainly consists of four components:

1. X-ray source emitting X-rays. The parameter of the X-ray system, physical filter and scan parameter determine the quality of the generated images. They are mainly dependent on material type and research issue. The emitted X-rays are attenuated by the object depending on the density and thickness of the material.

2. Rotating sample stage with mounted specimen. The sample stage is rotating stepwise through 360 degrees. One image is taken at every position.

3. Detector. The detector registers the attenuated X-ray and converts it into a twodimensional X-ray image.

4. Computer system. The three-dimensional volume data set is computed by the use of different algorithms.

Figure 1 shows the arrangement of the main components of the CT-system for nonmedical purposes.

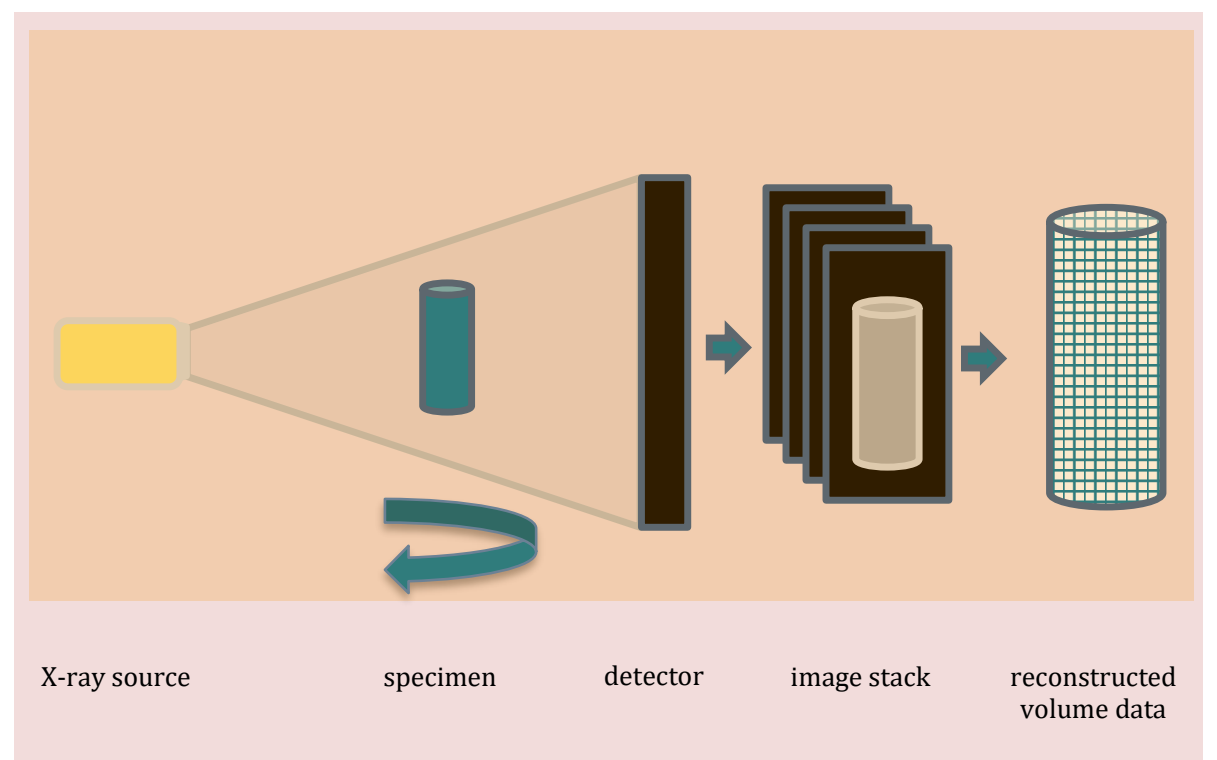

Figure 1: Principle of a CT-system 
For the scientific issues studied at the Bauhaus-Universität Weimar the GE "nanotom $m$ research I edition" is used.

Figure 2 shows the device and the opened measuring cabin.

The main features of the system are

- temperature stabilized digital detector (3.072 x 2.400 pixels)

- granite-based manipulator for high stability

- parameter:

- max. voltage

- min. voxel size

- geom. magnification

- max. sample diameter

- max. sample height

- max. sample weight

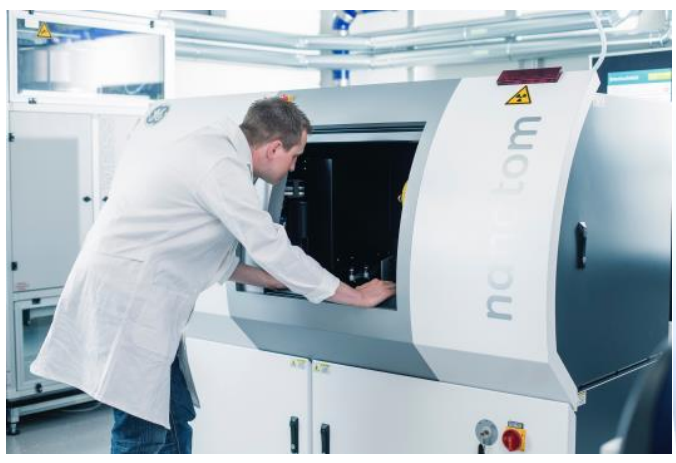

$180 \mathrm{kV}$

$<300 \mathrm{~nm}$

$1.5 \mathrm{x}-300 \mathrm{x}$

$240 \mathrm{~mm}$

$250 \mathrm{~mm}$

$3 \mathrm{~kg}$

Figure 2: CT-system nanotom m used at the Bauhaus-Universität Weimar

The achievable minimum voxel size mainly depends on material density and thickness. To roughly calculate the voxel size the longest path the X-rays take trough the specimen is divided by 1000 . Therefore, the smaller the specimen the smaller the achievable voxel size is [12]. Often the size of the specimen is a compromise between getting a high resolution and having a representative size of specimen.

\section{SELECTED EXAMPLES}

\section{CT for Understanding Complex Damaging Chemical Processes - ASR in Concrete}

In order to understand the underlying processes of alkali-silica-reaction (ASR) it is crucial not only to analyse 2-dimensional images from microscopy but also to observe the phenomena in 3-dimensional datasets. In [8] cracks as a result of damage caused by ASR are analysed by CT. The next step in understanding the underlying processes is to have a closer look at the microscopic structure of the ASR-gel. The main problem is the correlation between achievable voxel size and sample size. 
To detect the microscopic structures of ASR-gel, a very small sample size is needed. To obtain a representative part of sample which also contains a pore with ASRdeposits the specimen size is reduced stepwise. The smaller the object size the smaller the voxel size and the better the detail detectability is.

Figure 3 the effects of the reduction of the sample size on the resolution and the resulting voxel size respectively are shown.

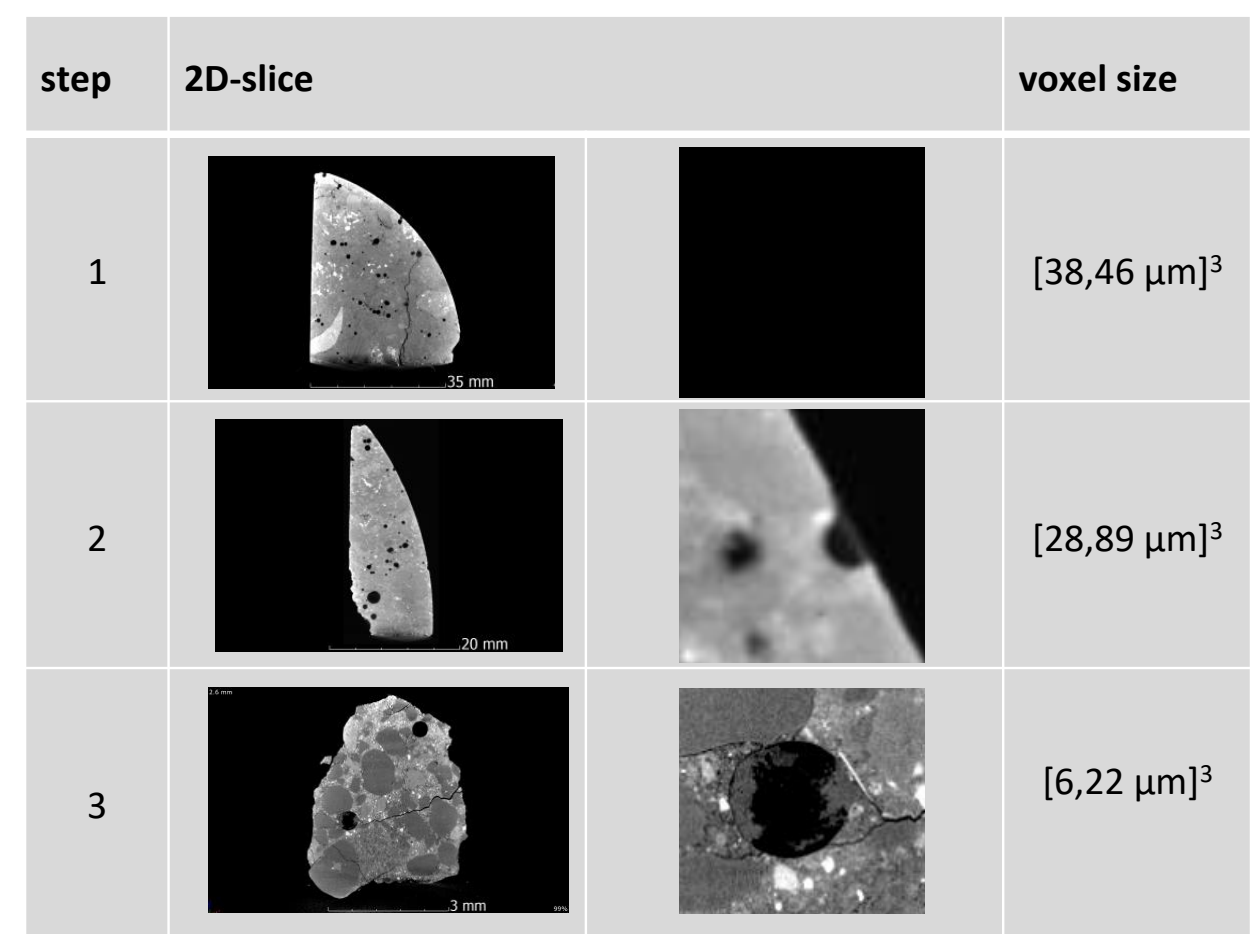

Figure 3: Effects of reducing object size

To analyse the structures of the ASR-gel deposit inside the pore clipping tool of the visualisation software was used. The specimen is virtually cut open to get a view inside the selected pore. Figure 4 shows the rendered and clipped specimen. On the left side, there is a classical rendering algorithm (Isosurface) used with the same grey value for every voxel. Density differences are not displayed. In order to give the object a 3dimensional appearance, virtual light sources are applied and shadows generated. Information about differences in density is included in the dataset.

There are also rendering algorithms which allow visualization of differences in density by varying the grey values of the voxels, for instance Phong-Shading. In case of irregular structures, it is not the method of choice because the human eye struggles in distinguishing between differences in density and rendered shadows. To get the maximum information out of the data false-colour rendering is used, like demonstrated on the right (Figure 4). Here every grey value is assigned a corresponding colour. Now it is much easier to distinguish between differences in density and rendered shadows. 

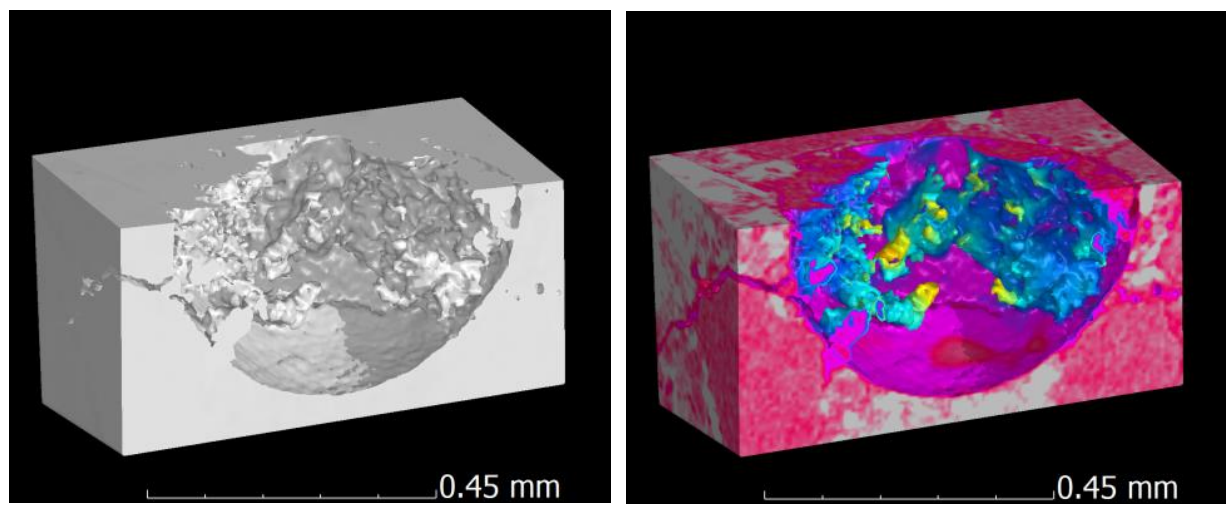

Figure 4: Inner structure of pore (classical rendering vs. false-colour rendering)

\section{CT for Geotechnical Purposes - Segmentation of Sphere Packing}

The research of characteristics and behaviour of soil structures, especially suffusion in case of water flow is done by combining laboratory experiments and numerical simulations. The main question of interest is to determine how the positions of the spheres (i.e. particles) change due to the flow of water through the particle packing. In order to reduce the amount of data, spheres are used to represent the particles of the soil. Otherwise it would not be possible to do the simulations in a finite time or with an adequate number of elements. To verify the simulation results, experiments with glass spheres are carried out. Computed tomography is used to determine the structure of sphere packing before and after the experiments.

Because of the high number of elements, it is insufficient to match the two datasets manually. It is necessary to automatically locate and segment the spheres in the CTdatasets.

Segmentation means the process of generating connected regions of voxels belonging to the same object. There are different methods to segment CT-datasets. [12] These methods are divided into five categories: pixel-based, edge-based, region-based, template-based and texture-based. The selected method depends on different factors like the quality and quantity of data, the power of the used computer, the available time, the image contrast, the object structure and texture, the resolution and the signalto-noise-ratio.

In [12] different methods are presented, with the help of which it is possible to evaluate the quality of the different segmentation algorithms. In the experiments presented here, we concentrated on the quality of segmentation of particularly critical areas.

Besides the conventional algorithms mentioned above, the spectrum of possibilities given by the software VG Studio MAX was used in this study. As with the other methods the segmentation results will improve with the image quality is. The original data sets used for the flow experiments contained a large number of spheres and also artefacts as shown in Figure 5. 

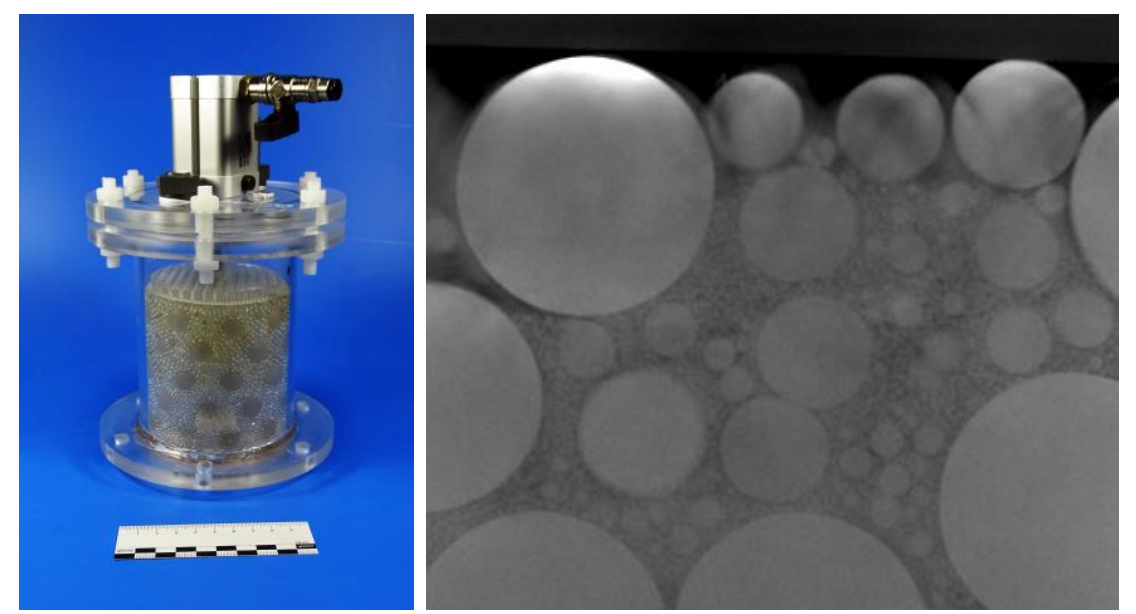

Figure 5: left: Part of the experimental setting containing the spheres right: Detail of a reconstructed cross-sectional image

Therefore, a smaller sample was used to test the methods for automated segmentation. Figure 6 shows the rendered scene. Details of the reconstruction process and filtering steps will not be discussed.

Figure 6 (left) shows the rendered scene. To get a free view of the spheres the container is hidden by adjusting the threshold for transparent materials. Contrast, resolution and detail detectability seem to high quality as can be seen at the cross-sectional images (right).

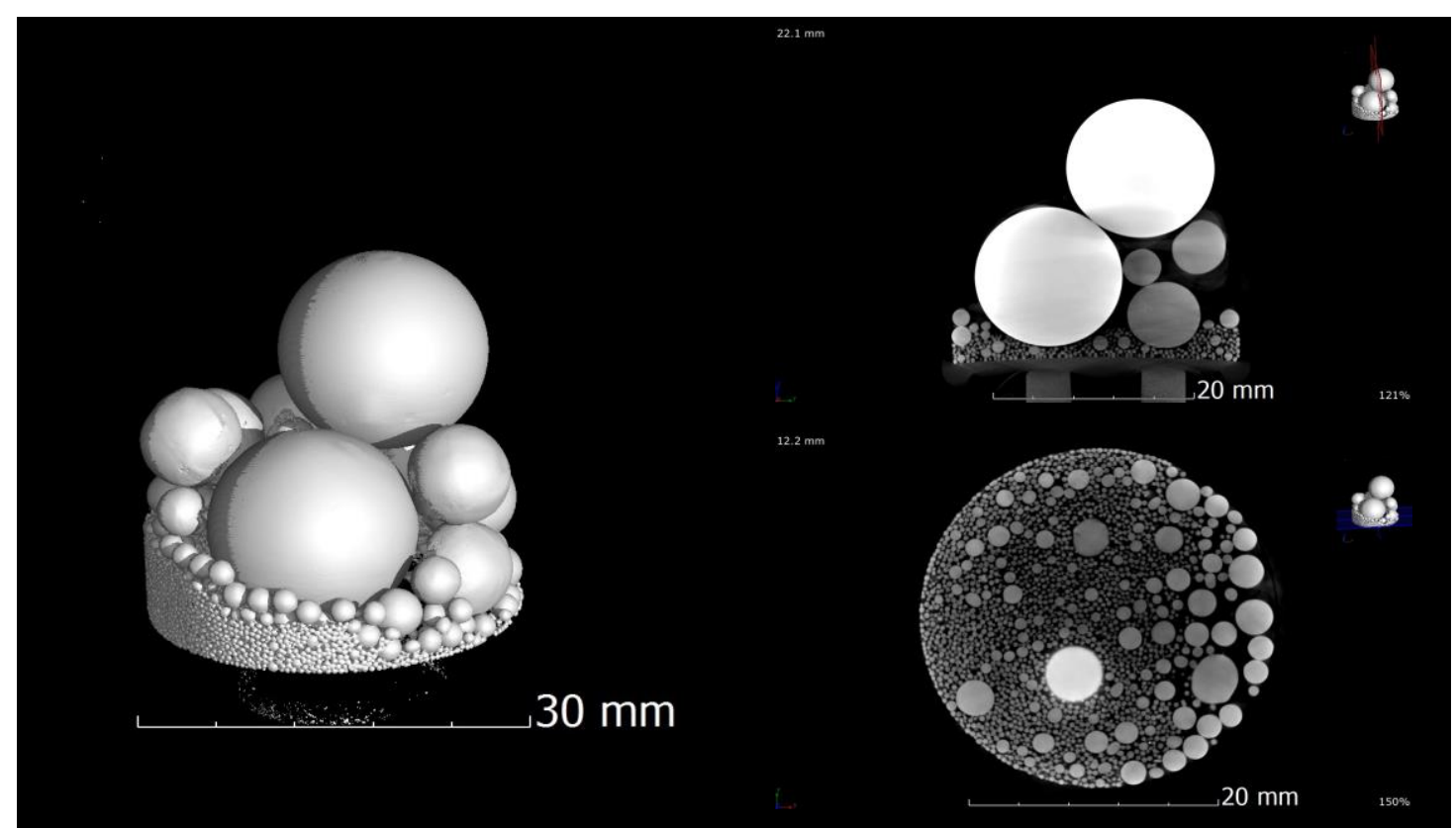

Figure 6: Reconstructed scene with hidden container 
Figure 7 shows the scene after automated segmentation. For this purpose, an additional commercial software module is used. Because the interface between two spheres is very thin and often low in contrast care must be taken when selecting a suitable segmentation algorithm or software module.

The big spheres were segmented as expected. The segmented spheres match the original spheres very well as can be seen in Figure 7. The algorithm found 9908 spheres in 4 sizes. The spheres are colour-coded according to their diameter. Table 1 shows size, colour and number of detected spheres.

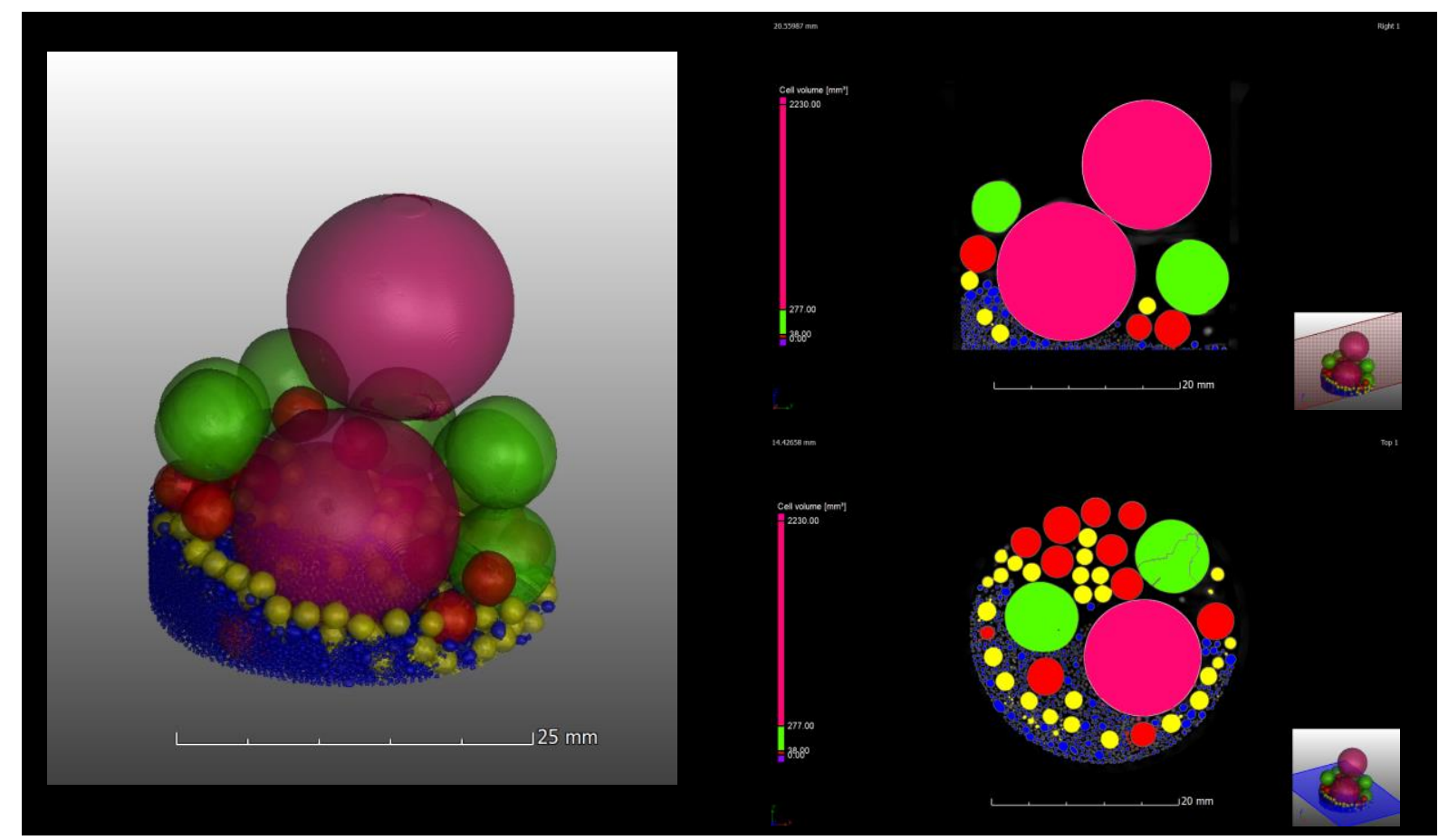

Figure 7: Segmented spheres colored by cell size

Table 1: Size colour and number of automated segmented spheres

\begin{tabular}{|c|c|c|}
\hline size (diameter) $[\mathrm{mm}]$ & color & number of detected spheres \\
\hline 16 & blue & 2 \\
\hline 8 & yellow & 7 \\
\hline 4 & red & 19 \\
\hline 2 & red & 114 \\
\hline$<2$ & red & 9766 \\
\hline
\end{tabular}

It can be observed that the bigger spheres were easier to segment by the software. The smaller the spheres are, the higher the probability of being considered as a part of another sphere is. 
Figure 8 shows under-segmented spheres, which means that several smaller spheres are combined to one larger sphere. The surface is not as smooth as expected.

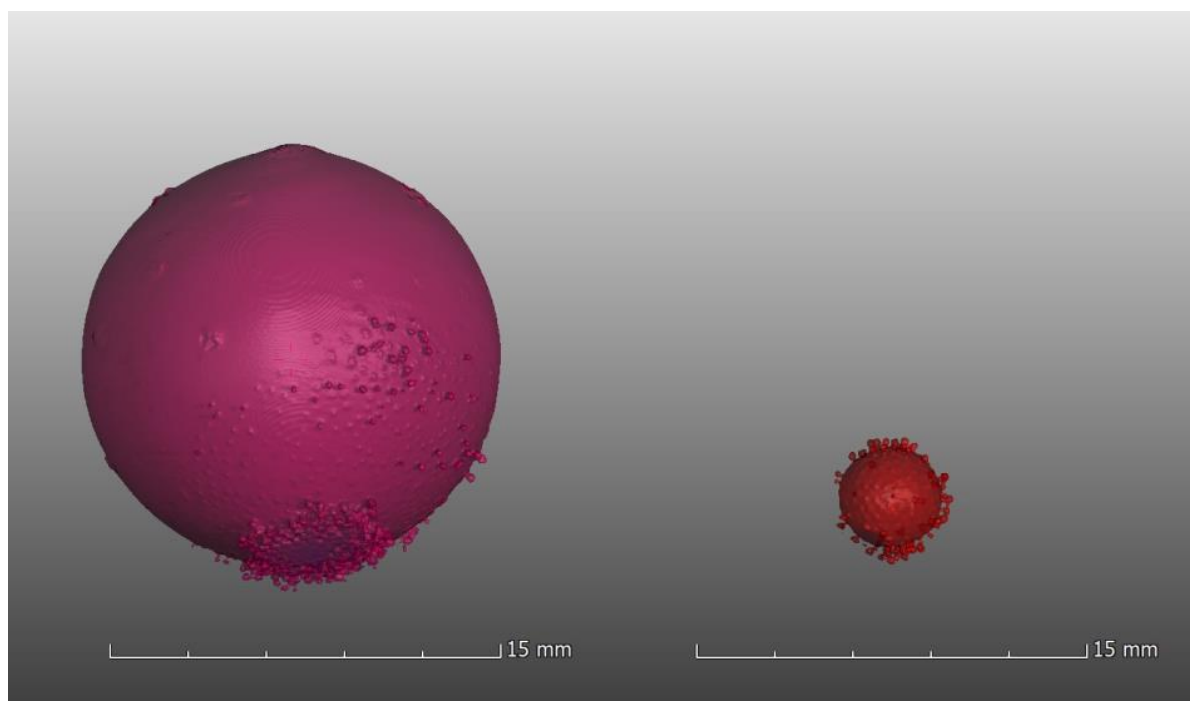

Figure 8: Examples for automated segmented spheres (under-segmentation)

Sometimes the spheres are divided into 2 or more parts by the software. This effect is called over-segmentation and is shown in Figure 9.

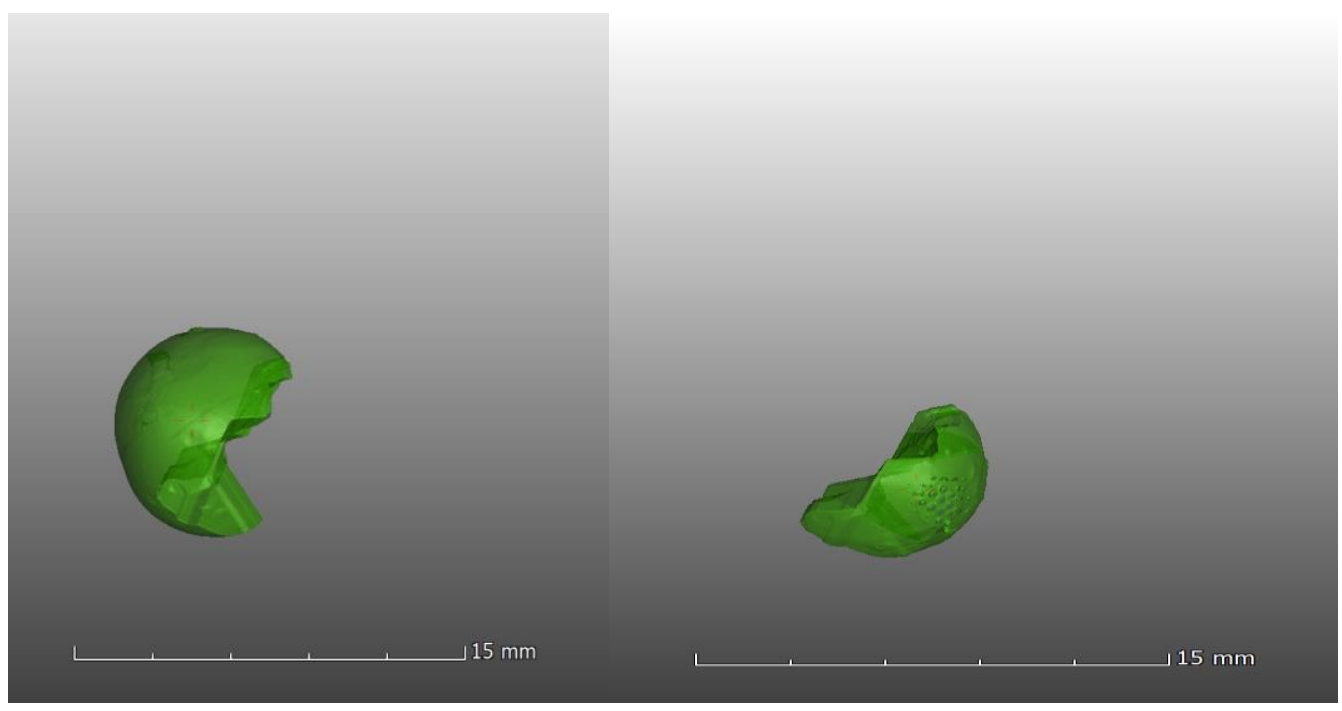

Figure 9: Sphere divided in 2 parts by segmentation algorithm (over-segmentation)

To get an overview of the number of spheres and their size distribution the automated algorithm works well. It segments the data set into spheres and determines different properties of every single element, such as the position, volume and sphericity. However, in order to use the data to validate numerical experiments more accurate segmentation is needed. 
Another approach of more accurate segmentation is to use the information that the elements the algorithm has to find are all spheres. The software needs only two or three points near the surface and then it defines the best fitting sphere considering the contrast between material and air. The method is very accurate but time consuming because the above-mentioned points on the object surface need to be selected manually. For instance, applying this method to the beforehand discussed example and assuming that five seconds are needed to define one sphere by clicking three points by hand, 14 hours are needed to detect 9908 spheres.

Figure 10 shows the same specimen segmented by this semi-automated algorithm. Only a part of the spheres is segmented to show how this method works. To be able to investigate sample sizes relevant for research purposes, further work an automation is necessary.

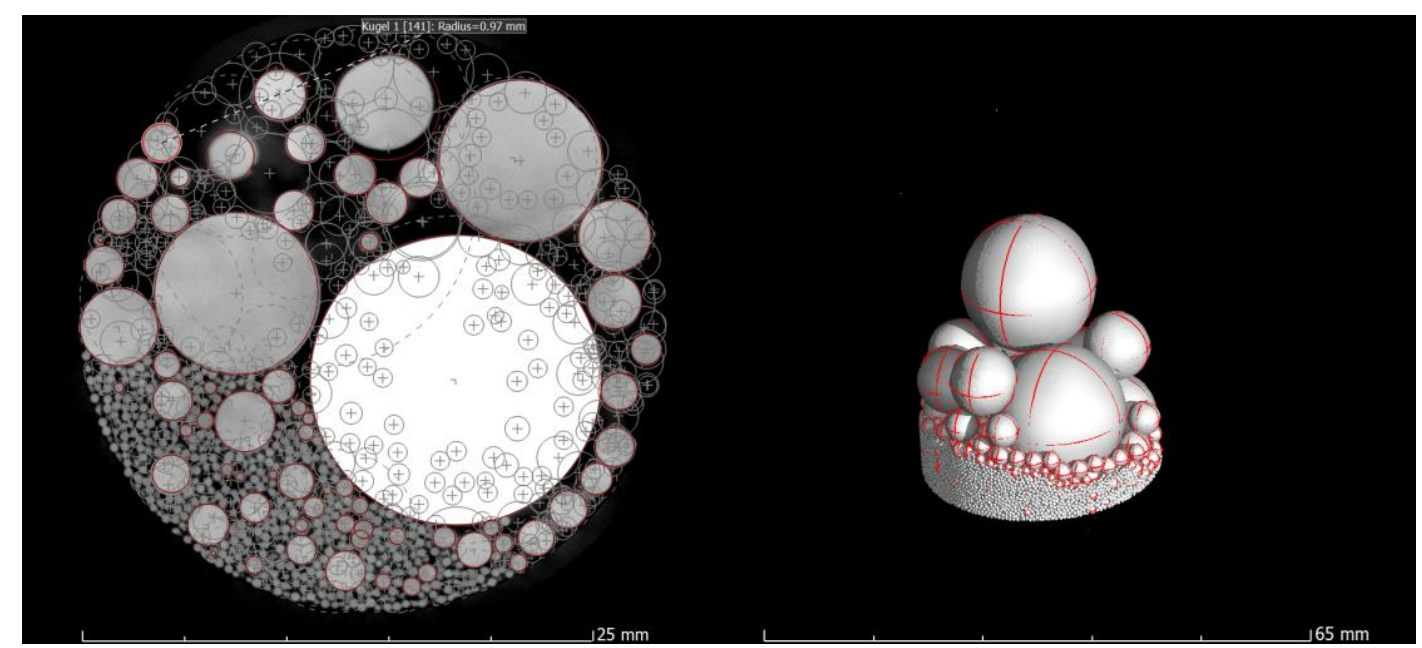

Figure 10: Partially segmented specimen with algorithm using geometry information

The method was also tested on a bigger specimen with more spheres and more artifacts. Some of the spheres were not perfectly circular. The algorithm wrapped the best fitting sphere around the elements. Figure 11 shows such a case.

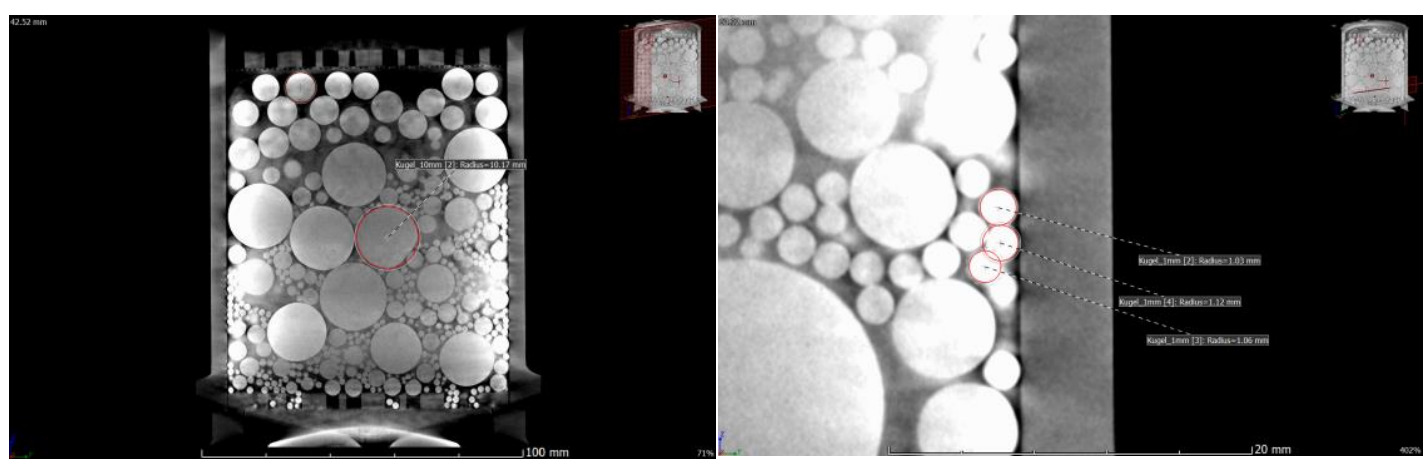

Figure 11: 2D slice (specimen container with spheres). Imperfect sphere wrapped in best fitting spheres. 
It can be shown that the deviation caused in imperfect spheres and artefacts in this method is smaller than the deviation caused by over- and under-segmentation in the previous shown method.

\section{CT for Geotechnical Purposes - Segmentation of Sand}

To analyse real soil structures, also scanning of sand and gravel is possible. In the following example, Pakistani sand was analysed by CT. The particular interest was in determining the sphericity of the particles and the grain size distribution.

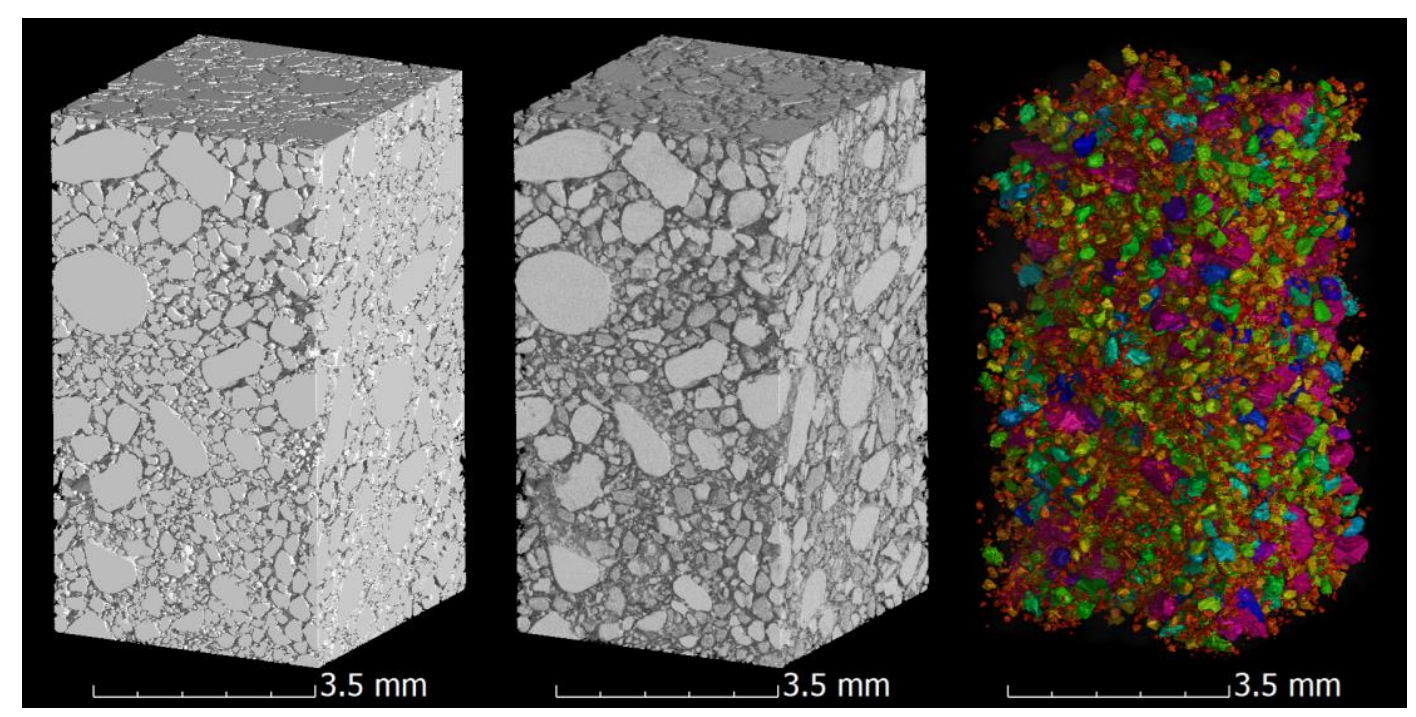

Figure 12: Different 3D-Renderings and segmented coloured sand grain

The same automated algorithm was used like in the case of the glass spheres. Here the problem with under-segmentation was not observed. The problem with oversegmentation also occurred in particularly cases.

The frequency distribution of the sphericity was as expected. The results of the segmentation of the CT-data were compared to the results of photo-optical analysis. 


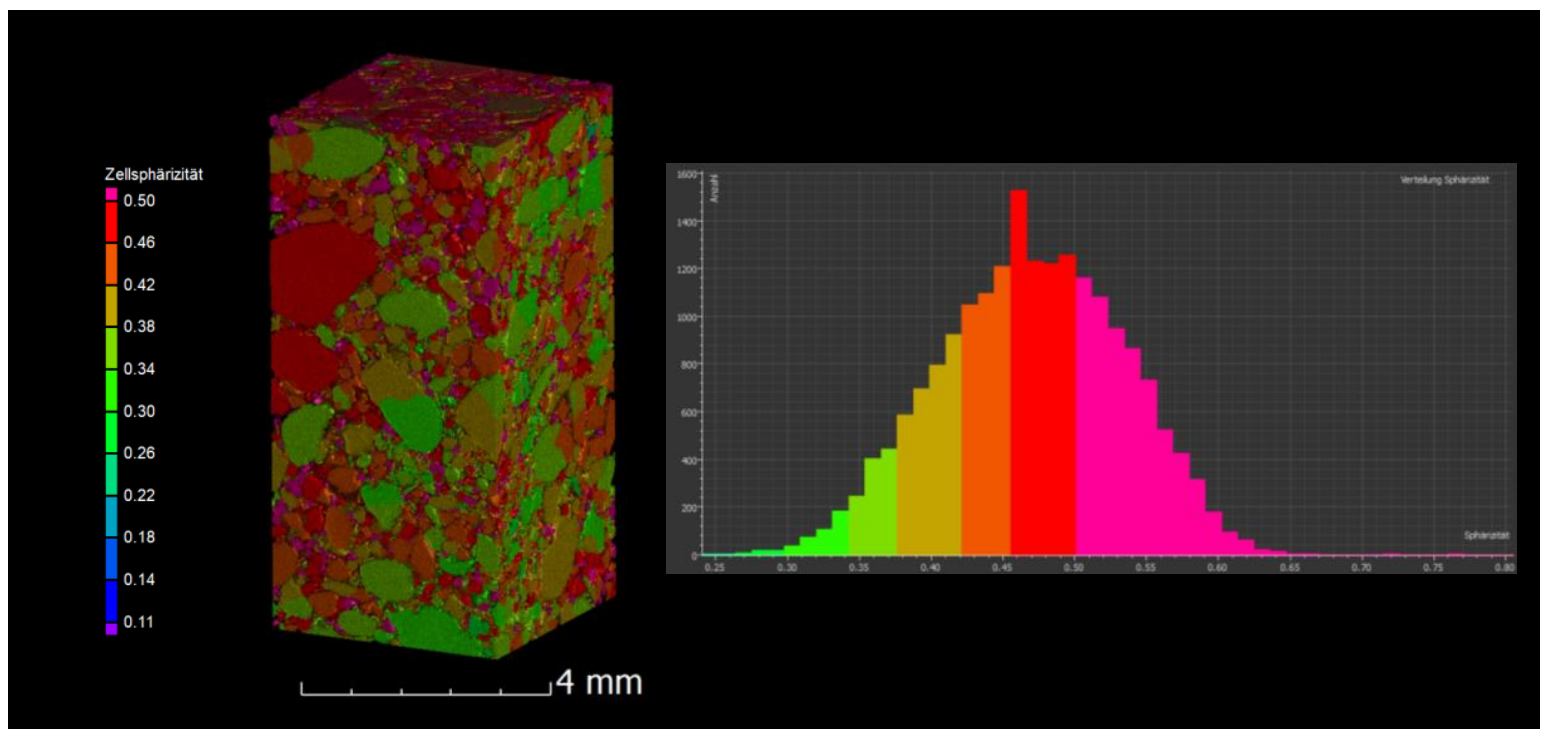

Figure 13: Sphericity of grain - coloured scene and frequency distribution

\section{CONCLUSIONS}

The selected examples have shown that computed tomography is a useful supplement to conventional methods of microstructure analysis of building materials. With the CTsystem used in this study a wide range of research questions regarding 3D-analysis of building materials can be covered and additional information on different scales can be generated.

\section{REFERENCES}

[1] Jacobs, P.; Sevens, E.; Kunnen, M.: Principles of computerized X-ray Tomography and Applications to Building Materials. Science of The Total Environment 167, 1-3 (1995), pp. 161 - 170

[2] Ruiz de Argandona, V. G.; Rey, A. R.; Celorio, C.; Suarez del Rio, L. M.; Calleja, L.; Llavona, J.: Characterization by computed X-ray tomography of the evolution of the pore structure of a dolomite rock during freeze-thaw cyclic tests. Physics and Chemistry of the Earth, Part A: Solid Earth and Geodesy. 24, 7 (1999) pp. 633 - 637

[3] Bentz, D. P.; Quenard, D. A..; Kunzel, H. M.; Baruchel, J.; Peyrin, F.; Martys, N. S.; Garboczi, E. J.: Microstructure and transport properties of porous building materials. II: Three-dimensional X-ray tomographic studies. Materials and Structures 33, 3 (2000) pp. 147 - 153

[4] Helfen, L.; Dehn, F.; Mikulik, P.; Baumbach, T.: Three-dimensional imaging of cement microstructure evolution during hydration. Advances in Cement Research, 17, 3 (2005) pp. 103 - 111 
[5] Koster, M.; Hannawald, J.; Brameshuber, W.: 3D Simulation of Moisture Transport in Porous Building Materials. In: Transport in Concrete: Nano- to Marcrostructure. Proceedings of 5th International Essen Workshop, TRANSCON 07, Duisburg-Essen, 2007, pp. 435-446.

[6] Elaqra, H.; Godin, N.; Peix, G.; Mili, M. R.; Fantozzi, G.: Damage evolution analysis in mortar, during compressive loading using acoustic emission and $\mathrm{X}$ ray tomography: Effects of the sand/cement ratio. Cement and Concrete Research 37, 5 (2007) pp. $703-713$

[7] Suzuki, T.; Ogata, H.; Takada, R.; Aoki, M.; Ohtsu, M.: Use of acoustic emission and X-ray computed tomography for damage evaluation of freezethawed concrete. Constrution and Building Materials. 24, 12 (2010) pp. 2347 $-2352$

[8] Weise, F., Voland, K., Pirskawetz, S., Meinel, D.: Analyse AKR-Induzierter Schädigungsprozesse in Beton. Beton- und Stahlbetonbau 107 (2012), Heft 12, S. $793-864$

[9] De Boever, W.; Derluyn, H.; Van Loo, D.; Van Hoorebeke L.; Cnudde, V.: Data-fusion of high resolution X-ray CT, SEM and EDS for 3D and pseudo3D chemical and structural characterization of sandstone. Micron, Volume 74, 2015, pp. 15 - 21

[10] Chung, S.-Y., Elrahman, m. A., Stephan, D., Kamm, P. H.: Investigation of characteristics and responses of insulating cement paste specimens with Aer solids using X-ray micro-computed tomograph. Construction and Building Materials 118 (2016) pp. 204 - 215

[11] Bacaicoa, I., Lütje, M., Sälzer, P., Umbach, C., Brückner-Foit, A., Heim, H.P., Middendorf, B.: Comparative investigation of two-dimensional imaging methods and X-ray tomography in the characterization of microstructure. Materials Testing 59 (2017), pp. 829 - 836.

[12] Cnudde, V.; Boone, M. N.: High-resolution X-ray computed tomography in geosciences: A review of the current technology and applications. EarthScience Reviews, Volume 123, 2013, pp. 1 - 17, ISSN 0012-8252.

[13] Kalender, W. A.: Computertomographie. Grundlagen, Gerätetechnologie, Bildqualität, Anwendungen. Erlangen: Publicis Corporate Publ 2006.

[14] C. Hadlich, F. Vogt, Osburg, A.: Methods for analyzing deposits in Pores using computertomography. 3rd International Conference on Tomography of Materials and Structures, 26th - 30th June 2017 Lund, Sweden

[15] Perciano, T.; Ushizima, D.; Krishnan, H.; Parkinson, D.; Larson, N.; Pelt, D.M.; Bethel, W.; Zok, F.; Sethian, J.: Insight into 3D-micro-CT data: exploring segmentation algorithms through performance metrics. Journal of Synchrotron Radiation, 2017. ISSN 1600-5775. 